\title{
Téoros
}

Revue de recherche en tourisme

\section{Retrouver Rhodes}

\section{Pierre Sintès}

Volume 29, numéro 1, 2010

Tourisme des racines

URI : https://id.erudit.org/iderudit/1024753ar

DOI : https://doi.org/10.7202/1024753ar

Aller au sommaire du numéro

\section{Éditeur(s)}

Université du Québec à Montréal

\section{ISSN}

0712-8657 (imprimé)

1923-2705 (numérique)

Découvrir la revue

\section{Citer cet article}

Sintès, P. (2010). Retrouver Rhodes. Téoros, 29(1), 37-45.

https://doi.org/10.7202/1024753ar

\section{Résumé de l'article}

À la fin de la Seconde Guerre mondiale, la communauté juive de Rhodes, présente dans l'île depuis plusieurs siècles, avait totalement disparue. Déportés ou enfuis, ceux qui s'appelaient les Rodeslis laissent derrière eux le quartier de la Djuderia dans l'ancienne ville fortifiée qui est alors investi par des habitants grecs orthodoxes. Les descendants de cette communauté disparue vivent désormais sur les cinq continents et ont conservé vivant le souvenir de cette origine. Depuis quelques décennies, certains d'entre eux sont même revenus à Rhodes pour de brefs séjours afin de retrouver les lieux de leurs origines personnelles ou celles de leur famille. Ce voyage sur un territoire souvent imaginé est le moment d'une grande émotion et d'une certaine introspection identitaire. Il peut parfois tout de même réserver quelques surprises à qui l'entreprend.
Ce document est protégé par la loi sur le droit d'auteur. L'utilisation des services d'Érudit (y compris la reproduction) est assujettie à sa politique d'utilisation que vous pouvez consulter en ligne.

https://apropos.erudit.org/fr/usagers/politique-dutilisation/ 


\title{
Retrouver Rhodes
}

\author{
Pierre SINTÈS \\ Maître de conférences \\ Université de Provence \\ sintes@mmsh.univ-aix.fr
}

\begin{abstract}
RÉSUMÉ: À la fin de la Seconde Guerre mondiale, la communauté juive de Rhodes, présente dans l'île depuis plusieurs siècles, avait totalement disparue. Déportés ou enfuis, ceux qui s'appelaient les Rodeslis laissent derrière eux le quartier de la Djuderia dans l'ancienne ville fortifiée qui est alors investi par des habitants grecs orthodoxes. Les descendants de cette communauté disparue vivent désormais sur les cinq continents et ont conservé vivant le souvenir de cette origine. Depuis quelques décennies, certains d'entre eux sont même revenus à Rhodes pour de brefs séjours afin de retrouver les lieux de leurs origines personnelles ou celles de leur famille. Ce voyage sur un territoire souvent imaginé est le moment d'une grande émotion et d'une certaine introspection identitaire. II peut parfois tout de même réserver quelques surprises à qui l'entreprend.
\end{abstract}

Mots-clés: Communauté juive, Rhodes, tourisme, patrimoine, Grèce.

Moins connue que celle des Sépharades de Thessalonique, la communauté juive de Rhodes partage pourtant avec l'ensemble des Juifs de Grèce l'histoire tragique de l'extermination d'une partie importante de ses membres au cours de la Seconde Guerre mondiale. Cet épisode a provoqué la disparition quasi totale de la communauté rhodienne des lieux où elle vivait depuis de nombreux siècles. Aujourd'hui, les descendants des Rodeslis composent des groupes de plusieurs milliers de personnes, établis essentiellement aux États-Unis, en Afrique du Sud, en Belgique, en Argentine ou en Israël, dont le souvenir d'une origine commune est encore des plus vivaces. Le terme Rodesli, qu'ils utilisent pour se nommer, est défini par Renée Hirschon (2005) comme étant «the Jewish inhabitants of the island of Rhodes and the original migrants, Rhodes (-origin) Jews, Sephardim. These are Sephardi Jews (Spanish speaking) who settled on the Aegean island of Rhodes following their expulsion from Spain in $1492 »$. Il s'agit en effet de l'autonyme couramment employé par les descendants des Juifs de Rhodes et qui signifie "rhodien" en turc. En français, ces membres se désignent indifféremment comme Rodeslis ou comme "Rhodiotes", nom dérivant cette fois-ci du terme grec.
Les descendants de ces Rodeslis sont nombreux à se reconnaître dans ce qu'il convient d'appeler une communauté : ils partagent un ensemble de représentations et de pratiques qui en témoignent a leurs yeux. Ils répondent ainsi a la définition que Jean-François Dortier donne à un collectif de «personnes unies par des liens de sociabilité assez étroits, une sous-culture commune et le sentiment d'appartenir a un même groupe» (Dortier, 2004). Cette communauté est définie par la transmission d'histoires de famille, d'une cuisine particulière, mais aussi dans la pratique d'une langue originale, le ladino, appelé aussi judezmo ou judéo-espagnol, qui marque toujours de son empreinte leur liturgie comme leur vie sociale. Il s'agit d'une langue dérivée de l'espagnol médiévale qui atteste le lien historique avec les communautés d'Al Andalus. Celle-ci comprend par ailleurs des emprunts nombreux aux langues des pays ou ces communautés ont vécu, ou encore à l'hébreu. Voir a ce sujet les travaux de David Bunis (2006) et de Thomas Harris (1994).

Parmi les différents moments qui permettent d'affirmer cette appartenance, le voyage en Grèce, plus particulièrement à Rhodes, est devenu un passage obligé, ou au moins largement désiré pour une bonne partie d'entre eux. Il peut avoir pour support un événement privé de la vie de famille (bar-mitsva 
d'un enfant, mariage d'un couple), mais aussi un simple séjour destiné, entre autres choses, à montrer aux jeunes générations les lieux d'origine de leur ascendance disparue ou déclinante.

Depuis le début des années 2000, l'institution d'un jour de commémoration de la mémoire des déportés en plein cœur de l'été a introduit une organisation plus poussée du voyage autour de cet événement et provoqué un regain significatif du nombre de visites. Voyage initiatique, pèlerinage familial ou commémoratif, ces différents aspects se mêlent désormais à cette occasion dans des pratiques qui tiennent souvent du voyage touristique : les intéressés fréquentent l'île sur leur temps de vacances. Le recueillement cède souvent la place au divertissement, puisque les lieux visités ont une signification particulière qui leur confère une forte charge émotive.

Ce texte a pour objet de présenter les modalités des voyages à Rhodes qu'effectuent les membres des familles juives dont les ascendants ont quitté l'île au cours du XX siècle. Ces «retours» paraissent être un trait intéressant de la remobilisation identitaire dont les Rodeslis font preuve à travers le monde. Surtout, ils nous donnent un bon point d'observation de dynamiques tout à fait contemporaines de réactivation identitaire par le biais de la mobilité géographique ainsi que d'évaluation de leur capacité à transformer les lieux qu'elles concernent. Ces conclusions s'appuieront sur une recherche menée depuis juin 2006 grâce au concours de l'École française d'Athènes, de l'UMR 6570 TELEMMe, du Centre de recherche à Jérusalem et de la Fondation du Judaïsme français, et avec l'aimable soutien à Rhodes de madame la Présidente Bella Angel-Restis et de madame Carmen Cohen. Ces différentes personnes m'ont permis d'effectuer plusieurs enquêtes à Rhodes auprès des visiteurs venus retrouver l'île de leur enfance ou de celle de leurs parents, mais aussi sur les lieux de leur installation actuelle (à Bruxelles, au Cap ou dans la métropole littorale israélienne d'Ashdod à Netanya). Au cours de ces huit séjours (d'une à trois semaines chacun entre 2006 et 2010), plus de 120 entretiens semi-directifs ont été effectués ainsi que de nombreuses observations in situ des modalités de transmission intergénérationnelle de cette mémoire.

\section{"Que reste-t-il de ma Djuderia?»}

Historiquement, les Juifs de Rhodes sont pour la plupart d'entre eux les descendants d'une communauté très ancienne, présente dans l'île depuis l'expulsion des Juifs d'Espagne en 1492 (Benbassa et Rodrigue, 2002 : 75 et suivantes). Cette longue présence s'interrompt brutalement au moment de la Seconde Guerre mondiale, plus exactement le 23 juillet 1944 quand l'armée allemande procède à la déportation de tous les Juifs présents à Rhodes vers les camps de concentration d'Auschwitz-Birkenau. Ils y furent exterminés pour la plupart dès leur arrivée (voir les travaux de Raoul Hilberg (2006) et, plus particulièrement pour la communauté juive de Rhodes, les ouvrages d'Ester Fintz-Menasce (2005) et d'Iskia Franco (1994)). Depuis cet événement, il ne reste pratiquement aucun représentant de cette communauté à Rhodes de manière pérenne, et seul son souvenir est entretenu parmi les différents groupes de ses descendants à travers le monde.

\section{Les cadres du souvenir}

Qu'il s'agisse des survivants de l'holocauste et de leur famille, ou bien des branches qui ont quitté l'île avant la guerre pour trouver une meilleure vie en migration, l'ensemble des Rodeslis rencontrés lors de ces différentes enquêtes sont apparus affectés d'un discours mémoriel dans lequel l'île de Rhodes tient une place centrale. (À propos de l'histoire de la dispersion des Juifs de Rhodes, voir les travaux de Renée Hirshon (2005) et de Marc D. Angel (1980), mais aussi le premier compte-rendu de cette enquête consacrée aux modalités de la revitalisation de cette mémoire à Rhodes, qui présente des éléments sur les différentes branches de la diaspora rhodienne à travers le monde (Sintès, 2010).) Ce lieu est présenté, plus que l'Espagne ou le biblique pays de Canaan, comme celui des racines de la communauté, métaphore assumée par exemple par le titre de l'ouvrage de Vittorio Alhadeff Le chêne de Rhodes. Cet ouvrage se présente comme une autobiographie qui raconte les tribulations d'un Juif de Rhodes ayant échappé à la déportation en partant pour l'Italie, puis vers le Nouveau Monde. Celui-ci finira sa vie à Buenos Aires après avoir revu l'île de son enfance une dernière fois (Alhadeff, 1998).

Plus particulièrement, la vieille ville médiévale, ceinte dans les murailles construites par les chevaliers de l'ordre de Saint-Jean de Jérusalem au XIV ${ }^{e}$ siècle, cristallise ce souvenir, car le quartier juif historique s'étendait dans sa partie orientale que l'on nommait alors la Djuderia. En son sein, la vie de la communauté est décrite comme ayant été parfaitement harmonieuse. Les bonnes relations qui y auraient prévalu sont souvent mises en avant : "Tout le monde connaissait tout le monde», et la concorde et l'entraide régnaient entre ses membres comme s'il s'agissait «d'une grande famille». C'est dans tous les cas l'image qui en a été transmise à leurs descendants qui la reprennent unanimement. L'existence d'institutions scolaires dispensant un enseignement en français (l'Alliance israélite universelle est installée à Rhodes à partir de 1899) ou en italien (au moment de l'occupation italienne de Rhodes entre 1912 et 1945), le caractère florissant de certains établissements commerciaux, l'ouverture d'un collège rabbinique en 1928 sont autant de sources de fierté pour ses descendants. Ces différents éléments leur permettent en effet d'affirmer que, sans être l'une des plus nombreuses de Méditerranée orientale ${ }^{1}$, leur communauté d'origine n'en était pas moins l'une des plus avancée en termes de vie communautaire : «ils étaient plus soudés qu'en Israël», comme nous le dit une jeune femme pour résumer la force de ce lien. Avant la guerre, les Juifs de Rhodes sont aussi décrits comme étant d'une très stricte observance religieuse. C'est à cette pratique que l'on attribue l'autre surnom du quartier juif de la vieille ville : la Chica Yérushalaim, la petite Jérusalem (Fintz-Menascé, 1991). Enfin, les relations avec les autres communautés religieuses de l'île, tout du moins avec les Italiens et les Turcs, sont aussi décrites comme pacifiques et respectueuses : au moment de sauver les Sefer-Torah des mains des Allemands, ne les a-t-on pas confiés au mufti de la ville?

L'évocation de cette vie paisible se mêle pourtant bien souvent avec la douleur du souvenir. Ainsi, le caractère 
idyllique des récits concernant la période d'avant la guerre est encore renforcé par le fait que Rhodes y apparaît comme un paradis perdu. Le contre-point de la déportation alimente encore la nostalgie des temps qui l'ont précédée, car le terme qui leur a été mis est un acte des plus barbares. «Nous vivions ici tranquillement, c'était un petit paradis. Nous étions à l'abri des horreurs du monde et de la guerre», dit l'une des survivantes de la Shoah. L'atrocité de cette disparition, l'indiscutable injustice qu'elle recèle, fait planer sur les rues de la vieille ville tristesse et mélancolie : «J’y entends la voix de mes parents », poursuit cette même personne. Ce lieu n'existe plus aussi par la faute des Grecs qui ont désormais pris place dans la ville médiévale et qui l'ont transformée en un espace entièrement dévolu au tourisme. "C'était le paradis. Ce que vous voyez aujourd'hui n'a plus rien à voir avec ce que c'était ", affirme un nonagénaire parti de Rhodes juste avant la guerre et aujourd'hui établi à Bruxelles. Ce décalage engendre aussi chez lui un certain ressentiment : "On vivait dans une ambiance saine et sainte. Maintenant, on a tout perdu. On se sent sans rien. On est devenu des étrangers, des Rashaïm [misérables], depuis que les nazis nous ont fait tout ce mal. Ils nous ont fait sauter en morceaux. Nous avons ici [en Belgique] tout ce que nous voulons, mais à l'intérieur de nous, tout est brulé. Nous ne pouvons pas retrouver ce que nous avons perdu. Nous n'avons plus rien». C'est ce même sentiment de désolation que l'on retrouve dans les premières minutes d'un documentaire réalisé en 1995 par Diane Perelsztejn intitulé Rhodes Nostalgie, où la lecture d'un poème par son auteur, l'un des aînés de la communauté (Joseph D. Alhadeff), exprime les différents sentiments éprouvés face aux lieux du souvenir.

\author{
KE KEDO DE MI JUDERIA? \\ QUE RESTE-T-IL DE MA DJUDERIA? \\ De la kay ancha al datilar \\ De la kay ancha au datilar \\ de l'espejo a la Puerta de la Mar \\ De l'espejo à la Porte de la mer \\ por las Siete Kalejikas \\ Entre ces sept ruelles \\ Dando bueltas i Bueltesikas \\ Je tourne et je retourne \\ Del Kal Grande a la Turkeria \\ De la Grande synagogue jusqu'à la Turkéria, \\ Ke kedo de mi juderia? \\ Que reste-t-il de ma Djuderia? \\ De Judios kedo vasia \\ Elle est désormais vide de Juifs \\ Ke pekado y ke mansia \\ Quel dommage et quel manque cruel! \\ Ma yo siempre busko i digo \\ Mais toujours je cherche et je me dis \\ Un pariente ... un amigo \\ qu'un parent... qu'un ami \\ Puede ser se salvaria. \\ peut-être, a puêtre sauvé. \\ Ke kedo de mi juderia? \\ Que reste-t-il de ma Djuderia?
}

\author{
Kaminando komo un loko \\ Marchant comme un fou \\ No topi muntcho ni poko \\ Je n'ai trouvé ni un peu ni beaucoup \\ De los mios, por dolor, \\ des miens, quelle douleur \\ Mas ni uno! Ke malor... \\ pas même un seul, quel malheur! \\ Por la poka l'alegria \\ Et, pour ne nous laisser que peu de joies, \\ Nada kedo de mi Juderia \\ Rien n'est resté de ma Djuderia
}

Ce poème met en scène efficacement les sentiments qui assaillent le narrateur lors d'un voyage de retour à Rhodes et de la visite de l'ancienne Djuderia. Celui-ci est bien confronté à des lieux familiers, qu'il appelle par les noms de l'usage ancestral de son enfance mais, plus que le plaisir de les retrouver, ils lui évoquent essentiellement la perte des êtres chers d'une part, le temps qui passe et qui les transforme inexorablement de l'autre. Pourtant, malgré ces sentiments assurément douloureux, ce type de voyage dans l'île de Rhodes a été le fait d'un nombre important de personnes ayant échappé à la mort durant la guerre, ainsi que celui de certains de leurs descendants.

\section{De l'impossible retour au tourisme généalogique}

Ces lieux pourtant ne sont pas uniquement ceux d'une mythologie familiale ou communautaire. Régulièrement depuis la fin de la guerre, certains Rodeslis ont franchi les kilomètres qui les séparent de Rhodes. Les premiers retours ont concerné les survivants de la Shoah dès les années qui suivirent leur libération des camps de concentration. En général, ces retrouvailles avec Rhodes sont toujours associées à des moments de grande douleur et d'émotion compte tenu des atrocités subies pendant la guerre mais aussi de ce qu'ils retrouvaient sur place. Toute chose dans ce quartier si familier leur rappelait alors la vie heureuse et insouciante de leur passé, mais aussi le souvenir des proches disparus dans les camps. Cette Djuderia où ils avaient grandi n'est plus désormais que "peuplée de fantômes » comme ils sont nombreux à en témoigner. Les maisons occupées par des réfugiés venus d'autres îles ou des villages environnants, la famine qui sévit, tout les pousse à quitter à nouveau cet endroit où l'avenir n'est pas envisageable. À la différence de ce que Lewkowicz a pu observer à Thessalonique, avec la réinstallation durable de certains survivants dans la ville (Lewkowicz, 2000), ces premiers retours sont sans lendemain et, mises à part les familles Soulam et Soriano, aucun Juif originaire de Rhodes ne demeure sur place après la guerre.

Pour les décennies suivantes, il est difficile de reconstituer précisément les venues des Rodeslis dans l'île. Il apparaît pourtant que la baisse du coût des transports aériens - et la démocratisation du voyage de longue distance qui en découle - est à l'origine du regain des visites que l'on constate dans le courant des années 1960. À partir de cette période, les voyages concernent des types beaucoup plus variés de personnes. Les motivations changent aussi : on ne 


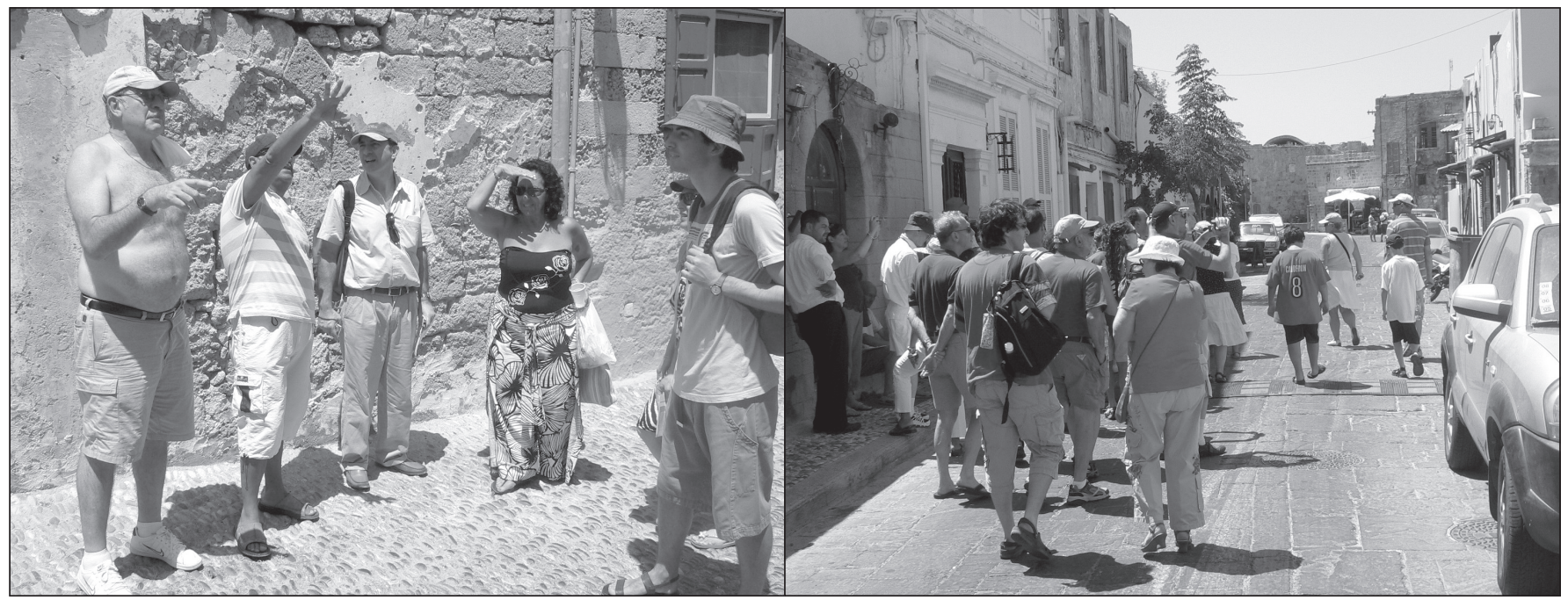

ILLUSTRATION 1 : Visiteurs rodeslis dans les rues de la vieille ville (photos : Pierre Sintès).

revient pas à Rhodes dans l'idée de s'y installer à nouveau ou d'y recouvrer des biens. Les parents de déportés de même génération viennent pour fréquenter, le temps d’un séjour, les ruelles de leur enfance, retrouver l'ambiance de la vieille ville : en deux mots, pour y «sentir leurs racines». Dans leur cas, le premier séjour s'effectue toujours dans les mêmes souffrances que pour les rescapés des camps de concentration. Pourtant, et malgré ces difficultés, le retour sur l'île semble s'imposer comme une sorte d'obligation morale. C'est une telle attraction qui s'exprime dans les lignes du récit autobiographique de Nisso Pelossof (2007: 9) : «Si je ressens une attache profonde pour cette région de Picardie qui m'a accueilli, la nostalgie de mes racines, de ma patrie l'île de Rhodes, - cette nostalgie est de plus en plus prenante au fil des années. [...] L'avenir se dessine progressivement pour moi d'un retour à Rhodes. C'est inéluctable.»

Implicitement, ce voyage est dû aux victimes de la déportation, mais aussi aux générations qui les ont précédées. Désormais il concerne aussi des personnes plus jeunes qui se rendent à Rhodes pour y découvrir le lieu de naissance d'un parent et surtout "pour y retrouver leurs origines, la terre de leurs ancêtres». Les jeunes gens qui en ont la possibilité, surtout venus d'Afrique ou des États-Unis, s'y rendent à l'occasion d'un voyage de noce ou en famille avec les enfants. Pour eux, ce voyage se teinte prioritairement d'une sorte de quête spirituelle ou identitaire. Il s'agit en quelque sorte d'un voyage destiné à résoudre la tension du discours nostalgique qui leur est imposé depuis leur enfance. "On aime tous Rhodes mais on n'y a jamais vécu. Ce sont nos parents qui nous ont «bourré le crâne» avec leur île», dit une quinquagénaire bruxelloise qui passe presque tous les étés à Rhodes depuis les années 1980 et dont la fille fait désormais de même. Plus prosaïquement néanmoins, pour les uns comme pour les autres, le voyage est indiscutablement l'occasion d'un séjour de vacances sous le soleil de Méditerranée. Il ne vise d'ailleurs plus exclusivement Rhodes. L'escale dans l'île est souvent incluse dans un circuit plus long comprenant différentes destinations, essentiellement Israël et l'Europe. Dans les récits des intéressés relevés à l'occasion des entretiens, on retrouve d'ailleurs de manière régulière l'évocation de la dimension touristique du séjour : la qualité de l'hôtel et des plages, la cuisine grecque que l'on découvre ainsi que l'accueil des populations locales sont très souvent mentionnés comme des aspects déterminants de sa réussite. La comparaison avec ce que l'on peut observer dans des périphéries moins touristiques de la Grèce est intéressante. Pour des raisons alors évidentes, ces visites estivales sur les lieux de leurs racines concernent bien moins les descendants des Juifs de la ville montagnarde d'Ioannina, dans le nord du pays, dont la communauté connaissait pourtant une dimension similaire à celle de Rhodes avant sa déportation et son extermination.

L'efficacité du voyage, en termes émotionnels, apparaît néanmoins dans les différents témoignages sans que l'on puisse remettre en question la sincérité du pèlerin. «Je pleurais tellement que je ne pouvais descendre de l'avion quand je suis arrivé à Rhodes", dit l'un des enfants de déportés. $\mathrm{Ou}$ alors «nous étions en croisière et nous nous sommes arrêtés à Rhodes. On a retrouvé un des autres passagers à la synagogue. On a compris qu'on était parents. On est tombé dans les bras l'un de l'autre et on a pleuré». Le voyage peut être aussi l'occasion de la production d'artefacts qui deviennent de nouveaux supports pour le souvenir. Il s'agit bien entendu des photos des lieux significatifs que l'on peut comparer aux souvenirs de vacances, mais parfois d'autres, plus originaux, viennent rappeler que Rhodes n'est pas une destination comme les autres. Ainsi, l'un de ces visiteurs a-t-il écrit le synopsis complet d'un film alors qu'il ne savait rien de ce travail avant : le simple fait d'être à Rhodes semblant lui avoir permis de composer, «comme si ce n'était pas lui qui écrivait», un texte techniquement parfait. Un autre a composé sur place des poèmes en ladino. Dans ces deux cas, la création est attribuée à l'inspiration du lieu. Implicitement, le rapport aux disparus est nettement suggéré dans les situations presque magiques que le voyage semble avoir engendrées. 
Plus anecdotique, entendra-t-on très souvent : «le ladino me vient plus naturellement quand je suis à Rhodes", comme si le simple fait de se trouver sur place pouvait décupler les compétences linguistiques des intéressés.

Pour ces différentes raisons, les Rodeslis sont les acteurs de ce qu'il convient d'appeler un "tourisme généalogique» tel qu'il a été observé ailleurs, par exemple chez les Juifs du Maghreb (Dakhama, 2005) ou d'Allemagne (Coles, 2003), chez les descendants d'Irlandais des États-Unis (Legrand, 2006b) ou les membre de la diaspora libanaise (Butler, 2003). En cela, ils présentent certaines particularités et leurs comportements sur place diffèrent indiscutablement de celui des nombreux autres touristes venus à Rhodes pour y passer des vacances sans que ce lieu ne leur évoque rien de leur vie personnelle ou de celle de leurs parents.

\section{Retrouver, fréquenter, organiser : de la mémoire à la réalité}

Malgré la diversité des personnes rencontrées sur place, force est de constater que les descendants des Rodeslis reproduisent à Rhodes certains comportements propres tout en étant eux aussi des vacanciers. En cela, ils passent des «vacances hybrides pour des personnes hybrides » comme le suggère Coles (2003 : 337), car ils ne peuvent être tenus ni pour de véritables touristes ni pour des autochtones comme les autres. S'ils fréquentent par exemple la ville médiévale, c'est surtout dans le but d'y retrouver des éléments attendus qui témoignent de la présence ancienne de Juifs à Rhodes. Il est intéressant de noter que, ces dernières années, ces pratiques ont été encadrées par des équipements ou des manifestations de mémoire venant offrir une structure d'organisation spatio-temporelle à ces séjours. Cette tendance participe clairement de l'institution d'un discours dominant sur l'histoire et l'actualité de la communauté rodeslie dont le voyage commémoratif est l'un des principaux instruments de diffusion. Cette normalisation de la mémoire s'appuie sur la mise en place d'un dispositif topologique (en voie de structuration) qui vise à donner une plus grande visibilité (et une plus grande légitimité) à la présence même temporaire de cette communauté dans l'espace public de la vieille ville de Rhodes.

\section{À la recherche de son passé}

Quel que soit son âge ou son origine, l'objectif déclaré du voyageur rodesli dans l'île de ses origines est toujours de voir, de constater, de connaître : c'est l'aspect «expérientiel» que souligne Coles (2003 : 329) pour d'autres lieux. Durant son court séjour (en général moins d'une semaine), il se rendra sans faute dans la vieille ville et dans le quartier de la Djuderia (voir illustration 1) même s'il n'y séjourne qu'exceptionnellement, préférant aux modestes auberges de la vieille ville les confortables hôtels à proximité de la plage. La dernière synagogue encore debout sur l'île, la Kahal Kadosh Shalom, qui date de 1577, est le lieu emblématique du séjour ainsi que les autres signes qui rappellent dans la vieille ville la présence d'une communauté juive. Au-delà de cet édifice, d'autres marques de ce passé sont en effet présentes dans l'espace public (voir illustration 2). Elles dessinent dans la vieille

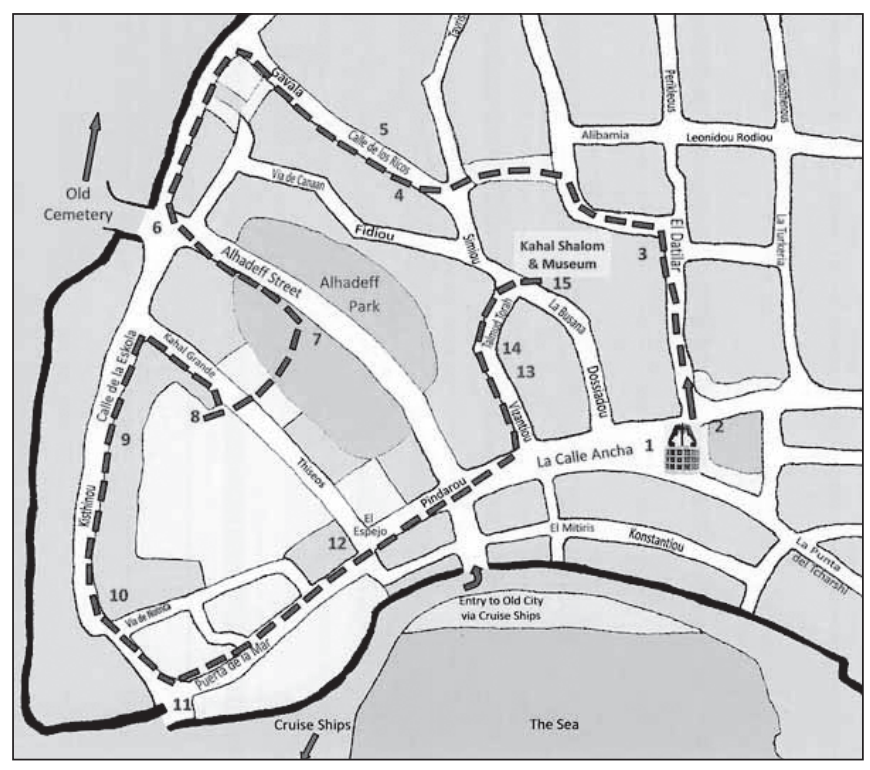

ILLUSTRATION 2 : Les «lieux à voir» en rapport avec la mémoire juive à Rhodes (source : http://www.rhodesjewishmuseum.org/juderia, avec l'aimable autorisation d'Aron Hasson).

ville une sorte de circuit qui opère un marquage identitaire de l'ancienne Djuderia. Celui-ci est alors emprunté par les visiteurs soucieux de retrouver la réalité du passé juif à travers des signes tangibles : inscriptions de donation apposées aux façades d'anciennes maisons, monuments ou plaques commémoratives. Cette pratique engage un processus de territorialisation qui passe tout d'abord par une première appropriation (que certains vivent comme une réappropriation) à travers la fréquentation des lieux. En dehors de la ville, plusieurs endroits peuvent être visités : le nouveau cimetière est une étape obligée pour la totalité d'entre eux tant il est directement lié avec la quête des origines. Mais d'autres lieux sont aussi recherchés car leur mémoire a été transmise dans les récits de famille.

Il n'est pas un Juif de Rhodes ou l'un de ses descendants qui ne se rende dans l'île sans aller sur les traces de la maison ou de la propriété de ses parents, de ses ancêtres avec les informations dont il dispose pour la retrouver. Ce type de visite peut le mettre en contact avec les habitants actuels de la vieille ville. La rencontre peut être tout à fait cordiale, et nombre de visiteurs ont été accueillis dans la vieille ville de manière sereine, comme cette famille du Cap qui s'est entendue dire "entrez, cette maison est plus la vôtre que la nôtre». D'autres racontent avoir ressenti une certaine inquiétude, une hostilité qui peuvent être liées à des malentendus, mais qui sont alors mises sur le compte de l'antisémitisme supposé des Grecs comme en témoigne le récit suivant :

Tous les Rodeslis cherchent leur maison quand ils viennent ici pour la première fois. Mais, tu sais comme c'est dur de parler de ces choses là ouvertement. Une fois, on se promenait dans les rues de la vieille ville et j'ai dit à David : "Regarde là! C'est la maison de ta grand-mère.» Le Grec qui l'habite a sorti la tête à 


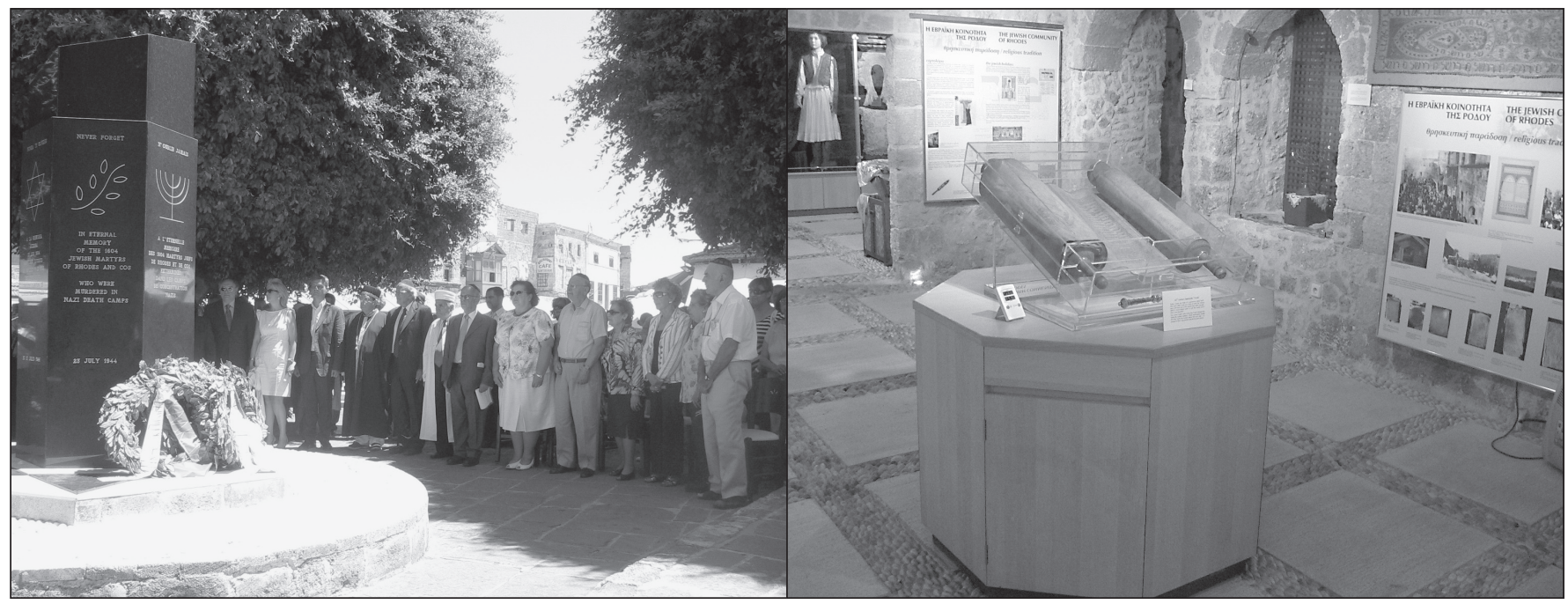

ILLUSTRATION 3 : Lieux et moment de plus grande visibilité de la communauté juive à Rhodes (photos : Pierre Sintès).

la fenêtre pour nous chasser... Ça leur a été facile de prendre l'histoire des gens sans rien payer. Souvent, on se fait refuser le droit d'entrer dans une maison même pour une minute, même pour prendre une photo.

Sans rien savoir de la situation tendue qui règne dans la vieille ville autour de la question immobilière entre les autorités municipales, les services archéologiques et les habitants, les visiteurs résument souvent ainsi leurs impressions : «Nous ne sommes pas les bienvenus à Rhodes.»

De manière générale cependant, il leur est toujours délicat de localiser précisément les différents éléments de cette mémoire, car ils ne font l'objet d'aucune mise en valeur par les autorités grecques qui leur préfèrent le patrimoine byzantin ou les traces du règne de l'ordre de Saint-Jean. Par conséquent, mais aussi en raison de l'implication des plus jeunes générations dans ces voyages, un nouvel acteur est en passe de s'imposer dans le jeu rhodiote. Il s'agit du «passeur de mémoire» qui, souvent rencontré à l'improviste, guide les pas des descendants, de leurs parents ou de leurs amis dans les ruelles de la vieille ville. Les premiers, les familles Soulam et Soriano déjà évoquées plus haut, étaient des résidents permanents, derniers témoignages d'une présence sépharade sur l'île. On les retrouve dans presque tous les récits relevés pour la période allant des années 1960 aux années 1990. Mais, depuis leur disparition, les passeurs sont désormais des intermittents qui vivent leurs retours réguliers à Rhodes sur le mode du témoignage. Les visiteurs qui les rencontrent s'en souviennent comme d'un fait marquant de leur séjour. En effet, ces personnes leur permettent de faire le lien entre le passé et le présent, leur donnent les clés de lecture nécessaires à révéler le paysage de la Djuderia sous celui d'une vieille ville désormais envahie de tavernes et de boutiques destinées aux touristes. Sous leurs bons auspices, se déroule une véritable initiation qui permettra au profane d'en apprendre plus sur les lieux, et sur la vie de la communauté dont il est le descendant. Mais ces passeurs peuvent aussi être des occasionnels, des personnes revenues à Rhodes pour la première fois et qui font profiter aux autres visiteurs de leur expérience. Il peut s'agir aussi de descendants de Rodeslis qui, très documentés sur l'histoire de leur famille, décident de partager leurs connaissances sur un lieu qu'ils découvrent parfois pour la première fois. Ce rôle non institutionnel est d'ailleurs transmissible et, une fois acquises différentes informations auprès des principales figures de la communauté, il n'est pas rare de voir un repeat visitor "faire la visite" pour ses amis, mais il veillera à s'effacer quand apparaîtra plus savant que lui. Cette connaissance des lieux est un savoir dont certains sont très fiers, surtout parmi les natifs, car "c'est un devoir de montrer ce qui a été ». La fierté peut parfois se muer en compétition, «certains disent qu'ils connaissent mais, en fait, ils ne savent pas vraiment. En fait il n'y a que moi qui sait où étaient les choses. Je suis la mémoire de Rhodes", explique l'un d'entre eux.

\section{La mise sur pied d'un premier dispositif d'accueil}

Depuis le début des années 2000, les choses n'en sont pas restées là. Sous l'impulsion de généreux mécènes, essentiellement une dame d'affaires rodeslie du Congo vivant à Athènes et un avocat de Los Angeles, petit-fils d'un Rhodiote arrivé aux États-Unis au début du $\mathrm{XX}^{\mathrm{e}}$ siècle, un mouvement de préservation de la mémoire de la communauté s'est fait jour. L'efficacité de ces différentes actions est en partie concomitante avec l'apparition d'outils au service de la préservation et de la diffusion du souvenir de l'origine commune. Les plus anciens sont des fascicules ou des lettres de liaison des communautés sépharades auxquels les Rhodiotes prennent part (La Lettre sépharade ou Los Muestros), mais il semble que ces nouvelles technologies de l'information et de la communication aient donnée un certain essor à ce type de support avec la création de sites internet spécialisés (voir illustration 2) et, depuis peu, d'un groupe de discussion Facebook qui rassemble quelques 500 personnes et d'un réseau aux très nombreuses entrées sur le site de recherche généalogique Geni. 
Un tel mouvement a conduit à l'amélioration considérable de la visibilité de l'histoire juive dans le quartier de l'ancienne Djuderia. En premier lieu, il a permis la rénovation en 2004, de la synagogue Shalom grâce à des fonds européens. Depuis sa restauration, elle accueille un grand nombre de touristes, mais elle est surtout en passe de devenir un point de ralliement pour les descendants de Rodeslis qui peuvent désormais se rendre à Rhodes à l'occasion des fêtes religieuses du calendrier hébraïque, ou pour y célébrer des événements de la vie familiale. Des bar-mitsvas et des mariages de personnes vivant aux quatre coins du monde y ont depuis été célébrés. En 2005 et 2006, ce sont 120 personnes du Zimbabwe, 200 de Dallas, qui sont venues assister à des mariages; au mois de juin 2007, plus de 100 personnes sont venues des Etats-Unis pour une Bar-Mitsva. Au cours des dernières années, la grande activité des mécènes associée à la forte demande des familles a conduit aussi à la mise à disposition du public d'un matériel de mémoire essentiellement constitué de photographies datant du début du $\mathrm{XX}^{\mathrm{e}}$ siècle, où chaque visiteur cherche à retrouver les ascendants de ses parents, de ses amis... et commente les traits de ressemblances physiques, confirmation à ses yeux du lien qui les unit dans une même communauté. Ce travail de mémoire entamé depuis les années 1990 a abouti en juillet 2007 à l'ouverture d'un musée, dans un espace attenant à la synagogue (voir illustration 3). Celui-ci permet l'exposition des objets recueillis dans le monde entier et de panneaux retraçant l'histoire de la communauté et expliquant sa disparition.

De la même manière, la mémoire des déportés est commémorée chaque 23 juillet dans la vieille ville, au beau milieu de la kaï ancha, la principale place de l'ancien quartier juif. Cette manifestation est accompagnée de toute une gamme d'activités qui conduisent à donner un certain présent au passé juif de la ville. Si, dans le reste du monde, la mémoire des victimes de l'holocauste est célébrée le 27 janvier (date de la libération du camp d'Auschwitz), il a été décidé ici de placer cette commémoration le 23 juillet, date de la déportation des Juifs de Rhodes. C'est un point de vue pratique qui a présidé à ce choix puisque Rhodes est une ville morte en hiver. Il est donc apparu plus pertinent d'organiser cette manifestation en été, au moment où elle peut être vue par le plus grand nombre de personnes, touristes comme habitants, mais aussi à une saison où l'île est plus attractive pour les Rodeslis du monde entier. Cet événement est en effet l'occasion de la venue de nombreuses personnes depuis tous les lieux de la diaspora. Les manifestations qui l'entourent donnent même lieu à un véritable pèlerinage/voyage organisé qui n'aurait rien à envier aux excursionnistes professionnels compte tenu du programme serré d'activités de groupe qui l'accompagne (dîners, visites, concerts), avec mise à disposition par les organisateurs d'autocars ou de guides le cas échéant. À travers un tel programme, la coloration touristique prise par ce retour aux sources se manifeste très clairement. La journée de la commémoration proprement dite débute par une cérémonie religieuse à la synagogue Shalom suivie de discours d'officiels. Les autorités grecques y sont présentes ainsi que certains représentants religieux de l'île (catholiques et musulmans - c'està-dire des autres minorités religieuses officielles présentes à
Rhodes). Cette cérémonie est suivie d'un dépôt de gerbes au monument commémoratif construit en 2002 au milieu de la kaï ancha. Ces deux moments ont un caractère interreligieux discret puisque les représentants des autres confessions ne prennent pas la parole. Il s'agit aussi d'un acte officiel de la république hellénique comme l'attestent les interventions des autorités ainsi que la présence de la fanfare municipale qui joue l'hymne national pour clore les dépôts des gerbes. Pourtant, de ce point de vue, un certain flou demeure qui confère aussi à cet événement les caractéristiques d'une commémoration privée dans laquelle l'entre-soi des visiteurs est partiellement mais efficacement conservé. Depuis 2007, les participants entonnent en effet l'Hatikvah après le dépôt de gerbes, et l'incompréhension des passants, voire des riverains est palpable. Ce double mouvement d'ouverture et de fermeture apparaît aussi dans le discours des autorités municipales et préfectorales grecques. Il faut dire qu'une telle manifestation n'est pas sans impliquer plusieurs types d'enjeux qui concernent le devenir de la vieille ville, comme la question des biens des déportés (Sintès, 2010).

On peut comparer une telle mise en valeur touristique du passé juif avec d'autres cas en Europe comme les «Routes de Sépharade» en Espagne, qui visent à promouvoir dans différentes villes de ce pays la mémoire des communautés juives disparues (Levy et Olazabal, 2003), ou bien les opérations menées dans le Comtat Venaissin depuis 1992 autour des communautés juives de Provence (Grange, 2003). Dans le cas de Rhodes, il est alors frappant de noter la grande originalité des modalités de cette revitalisation : les principaux acteurs y sont exclusivement privés et les autorités locales ne paraissent que très marginalement concernées. Pourtant, depuis quelques années, la mise en valeur de la vieille ville a fait l'objet de nombreuses interventions de la part des pouvoirs publics, s'appuyant sur le classement de ce site au patrimoine mondial de l'humanité en 1988 par l'UNESCO. Un tel décalage conduit à aborder la question de la difficile compatibilité de ce type de réactivation d'une "identité périphérique» avec le discours national grec, fondé depuis le XIX ${ }^{\mathrm{e}}$ siècle sur la Romiossini, c'est-à-dire sur la double identification à la langue et à la religion (Drettas, 2003; Herzfeld, 1995 : 41-42), qui tendrait à exclure les Juifs sépharades du groupe national pour des raisons aussi bien religieuses que linguistiques. Ceci est renforcé par la position particulière de Rhodes au sein de la construction de l'État grec moderne. En effet, l'île a été l'une des dernières entités à être rattachée à son territoire en 1947. De ce fait, le processus d'intégration de cet espace peut sembler inachevé alors que l'île occupe une position géopolitique particulière en raison de sa proximité avec la Turquie et de sa localisation très orientale en mer Égée. Le processus d'intégration est historiquement lié à l'homogénéisation des populations (Pierron, 1996). Or, à Rhodes, outre les rares descendants de la communauté juive historique ou les quelques Juifs venus de Grèce continentale après guerre, la ville principale connaît une importante communauté de Musulmans turcophones ainsi que de Catholiques romains. Ceci lui confère une dimension multiconfessionnelle rare en Grèce, dont la population est officiellement composée à 97,6\% de Chrétiens orthodoxes (Clogg, 2002). 
Dans un contexte idéologique où l'homogénéité linguistique ou religieuse a souvent été associée à la loyauté des populations, il est facile de comprendre la suspicion pouvant entourer la question des identités minoritaires. Ceci expliquerait aussi le faible intérêt des autorités publiques grecques et des habitants de la vieille ville pour la revitalisation de la mémoire de la communauté juive à Rhodes. C'est aussi l'une des raisons pour lesquelles, comme dans l'ensemble des régions dites stratégiques en Grèce, les étrangers n'ont pas eu le droit de posséder des propriétés immobilières jusque dans les années 1980, dissuadant ou empêchant de fait les rescapés de la Shoah ou leurs descendants de recouvrir leurs biens à Rhodes.

La différence paraît alors grande avec ce qui a pu être étudié ailleurs, par exemple dans le cas de la ville catalane de Gérone, où les élites locales revendiquent au contraire une filiation cachée avec la tradition juive. À travers cette comparaison, on comprend bien que la réalisation de ce type très particulier de tourisme, qui implique un rapport au territoire élaboré sur le mode de l'appartenance, «n'obéit pas seulement à des enjeux liés à la concurrence internationale pour attirer des visiteurs, mais elle met en jeu, sur le plan idéologique, des représentations de la nation, de son histoire et de la place des différents groupes qui ont participé à sa construction culturelle par le passé» (Levy et Olazabal, $2003: 65$ ). Ceci éclaire la dimension exclusivement exogène de la mobilisation que nous venons de décrire pour Rhodes, caractéristique qui engage la question de sa durabilité à moyen terme.

\section{Conclusion : tourisme durable ou feu de paille?}

À travers cette pratique d'un tourisme particulier, les Juifs de Rhodes ont pu retrouver les lieux de leurs origines. Ce mouvement témoigne une fois encore qu'un groupe en diaspora, ayant entretenu un fort sentiment d'attachement pour un homeland, peut devenir l'un des acteurs de son développement économique ou culturel. Le mot est ici entendu dans son sens premier, car ce groupe a été, à l'image des Juifs de l'Antiquité, dispersé de par le monde à la suite d'un événement traumatisant. Cette caractéristique est l'un des fondements de la définition d'une diaspora (Safran, 1991).

Dans le cas des Rodeslis, la mémoire de Rhodes semble avoir été renforcée par le sentiment d'une perte injuste ou irrémédiable, en raison des circonstances historiques de la disparition de la communauté pendant la guerre ainsi que de la suspicion actuelle des autorités grecques. Cette particularité, qui tient à la position doublement périphérique de l'île de Rhodes (dans l'espace pratiqué par les Juifs de Rhodes qui ne la fréquentent qu'exceptionnellement maintenant ou dans la construction du territoire grec), aurait longtemps soutenu le discours d'appartenance, créant aujourd'hui des conditions très favorables à la revitalisation de la mémoire juive sur place.

Pourtant, plusieurs aspects de cette mobilisation semblent indiquer qu'elle ne pourra pas se poursuivre. L'activité de fréquentation et d'appropriation de l'espace de la vieille ville à travers ces visites régulières, même si elle est présentée comme un moyen de sauvegarder le souvenir menacé de cette communauté, conduit en effet à désacraliser l'île par la banalisation du voyage. C'est ainsi que le séjour peut donner lieu à des déceptions devant la réalité d'un lieu si longtemps mythifié : «moi, la kai ancha, je croyais que c'était les champs Élysées » ou alors «je ne me souvenais pas que la fontaine était si petite», pour les plus âgées qui sont partis avant guerre... pour les plus jeunes : «j’en ai marre des mézzés, je veux un vrai plat!» ou alors «en Israël, il y a un vrai combat, ici ce ne sont que des pierres». Ces différentes remarques nous font percevoir combien la pratique du "paradis perdu» peut mettre un terme à la nostalgie que sa perte avait engendré, et qui en motive la visite.

Surtout, sans implication de la société locale, il est difficile de penser que ce type de visites pourra perdurer au-delà de la prochaine génération, car déjà s'éteignent ceux qui ont connu Rhodes pour y être nés. Pourtant, les autorités rhodiennes pourraient décider de valoriser cette forme de tourisme si différente de celui qui défigure leur littoral. Mais, dans cette perspective, les pouvoirs publics semblent plutôt se tourner vers des groupes dont l'expression identitaire est plus conforme à celle du pays. C'est le cas en particulier pour le tourisme des Grecs de la diaspora (Thanopoulos et Walle, 1988) qui, pour l'archipel du Dodécanèse, est promu depuis plusieurs années par l'association dodecanesian dream. À la différence des manifestations de la communauté juive, les autorités publiques, comme la municipalité de Rhodes et le TEDK (instance intercommunale) de l'archipel du Dodécanèse, participent activement aux commémorations concernant ce groupe, par exemple la première journée internationale de la diaspora dodécanésienne qui a eu lieu à Rhodes en août 2008. Lors de cette journée, aucune activité ne rappelait le passé multiconfessionnel de l'île, laissant entendre que les barrières de la religion, héritées du passé ottoman et du discours des nations, s'étendaient irrémédiablement aux structures d'une diaspora établie pourtant depuis plusieurs générations dans le monde entier.

\section{Note}

1 La communauté de Rhodes n'aurait jamais excédé 5000 personnes selon les estimations de Moïse Rahmani (2000) contre plus de 90000 à Salonique (Mazower, 2004).

\section{Bibliographie}

ALHADEFF, Vittorio (1998) Le Chêne de Rhodes, saga d'une grande famille sépharade, Paris : Paris-Méditerranée. 291 p.

ANGEL, Marc-D. (1980) Jews of Rhodes, the history of a Sephardic Community, New-York : Sepher-Hermon Press. 199 p.

BENBASSA, Ester et Aaron RODRIGUE (2002) Histoire des Juifs sépharades, de Tolède à Salonique, Paris : Le Seuil (coll. Point Histoire). 468 p.

BUNIS, David (2006) «Les langues juives du Moyen-Orient et de l'Afrique du Nord», DANS Le monde sépharade, sous la direction de Shmuel TRIGANO, p. 537-564. Paris : Le Seuil.

BUTLER, Richard (2003) «Relationships between Tourism and Diasporas : influences and Patterns ", Espace, populations et sociétés, $\mathrm{n}^{\circ} 2$, p. 317-325. CLOGG, Richard (2002) Minorities in Greece, aspect of a plural Society, Londres : Hurst. 203 p.

COLES, Tim (2003) «Diaspora and the Mediation of Vacationscapes: Some Lessons from Enticing Jewish-Americans to Germany», Espace, populations et sociétés, $\mathrm{n}^{\circ} 2$, p. 327-340. 
DAKHAMA, Hicham (2005) «La présentation de soi au pèlerinage de Rabbi Hahya Lakhdar (Maroc)", DANS Les pèlerinages au Maghreb et au Moyen-Orient. Espaces publics, espaces du public, sous la direction de Sylvia CHIFFOLEAU et Anna MADEUF, p. 39-58. Beyrouth : Ifpo.

DORTIER, Jean-François (2004) Dictionnaire des sciences humaines, Paris : Éditions Sciences Humaines. 875 p.

DRETTAS, George (2003) «Pour une anthropologie religieuse de la Grèce contemporaine», Messogeios, $\mathrm{n}^{\circ}$ 20-21, Herodotos, p. 21-41.

FINTZ-MÉNASCÉ, Ester (1991) Gli ebrei a Rodi, Milan : Guerini. 595 p.

FINTZ-MÉNASCÉ, Ester (2005) Buio nell isola del sole : Rodi 1943-1945, Florence : La Giuntina. 478 p.

FRANCO, Hizkia-M. (1994) The Jewish Martyrs of Rhodes and Cos, Harare: Harper Collins. 118 p.

GRANGE, Sophie (2003) «Le patrimoine juif comtadin. Un enjeu de développement local ?», Le tourisme de mémoire, Les Cahiers Espaces, $\mathrm{n}^{\circ}$ 80, p. 105-108.

HARRIS, Tracy K. (1994) Death of a Language: The History of Judeo-Spanish, États-Unis : University of Delaware Press. 354 p.

HERZFELD, Mickael (1995) «Les enjeux du sang, la production officielle des stéréotypes dans les Balkans : le cas de la Grèce», Anthropologie et Sociétés, vol. 19, n 3, p. 37-51.

HILBERG, Raul (2006) [1961] La destruction des Juifs d'Europe, Paris : Folio. $2400 \mathrm{p}$.

HIRSCHON, Renée (2005) «Jews from Rhodes in Central and Southern Africa», DANS Encyclopedia of Diasporas, Immigrant and Refugee Cultures Around the World, sous la direction de Melvin EMBER, Carol M. EMBER et Ian SKOGGARD, p. 925-934. Dordrecht : Kluwer Academic Publishers.

LEGRAND, Caroline (2006a) La quête de parenté. Pratiques et enjeux de la généalogie en Irlande, Canada : Presses universitaires de Laval (coll. Interculure). $168 \mathrm{p}$.
LEGRAND, Caroline (2006b) «Tourisme des racines et confrontations identitaires dans l'Irlande des migrations", Diaspora, histoire et société, $n^{\circ} 8$, p. 162-171.

LEVY, Joseph Josy et Ignace OLAZABAL (2003) «Les routes juives d'Espagne réveillent une mémoire oubliée. L'exemple de Gérone», Tourisme de mémoire, Les cahiers Espaces, n 80 , p. 59-65.

LEWKOWICZ, Bea (2000) «After the war we were all together», DANS After the war was over, Reconstructing the Family, Nation, and State in Greece, 1943-1960, sous la direction de Marc MAZOWER, p. 247-272. Princetown : Princetown University Press.

MAZOWER, Marc (2004) Salonica, City of Gosts (Christians, Muslims and Jews 1430-1950), Londres : Harper Collins. 525 p.

PELOSSOF, Nisso (2007) Nisso, d'une île à l'autre, Amiens : Encrage-Picardie. $191 \mathrm{p}$.

PIERRON, Bernard (1996) Juifs et Chrétiens de la Grèce moderne, histoire des relations intercommunautaires de 1821 à 1945, Paris : L'Harmattan (coll. Histoire et perspectives méditerranéennes). $271 \mathrm{p}$.

RAHMANI, Moïse (2000) Rhodes, un pan de notre mémoire, Paris : Romillat. $232 \mathrm{p}$.

SAFRAN, William (1991) «Diasporas in modern societies: myths and of homeland and return», Diaspora, vol. 1, n 1, p. 83-99.

SINTĖS, Pierre (2010) «Mi akodro de Rhodes, paroles d'une communauté juive de la mer Égée entre mémoire et actualité», DANS Les échelles de la mémoire en Méditerranée, sous la direction de M. CRIVELLO, Paris : Acte Sud/MMSH, p. 75 à 107.

THANOPOULOS, John et Alf H. WALLE (1998) «Ethnicity and its relevance to marketing : the case of tourism ", Journal of Travel Research, vol. 26, $\mathrm{n}^{\circ} 3$, p. 11-14. 